Europhysics Letters

PREPRINT

\title{
Stabilization of three-dimensional matter-waves solitons in an optical lattice
}

\author{
Marek Trippenbach ${ }^{1}$, Michae Matuszewski ${ }^{1}\left(^{*}\right)$ and Boris A. Malomed ${ }^{2}$ \\ 1 Physics Department, Warsaw University - Hoża 69, PL-00-681 Warsaw, Poland \\ 2 Department of Interdisciplinary Sciences, School of Electrical Engineering, Faculty of \\ Engineering, Tel Aviv University - Tel Aviv 69978, Israel
}

PACS. 03.75.-b - Matter waves.

PACS. 03.75.Lm - Tunneling, Josephson effect, Bose-Einstein condensates in periodic potentials, solitons, vortices and topological excitations.

PACS. 05.45.Yv - Solitons.

\begin{abstract}
We propose an experimentally relevant scheme to create stable solitons in a three-dimensional Bose-Einstein condensate confined by a one-dimensional optical lattice, using temporal modulation of the scattering length (through ac magnetic field tuned close to the Feshbach resonance). Another physical interpretation is a possibility to create stable 3D "light bullets" in an optical medium with a longitudinal alternating self-focusing/defocusing structure, and periodic modulation of the refractive index in a transverse direction. We develop a variational approximation to identify a stability region in the parametric space, and verify the existence of stable breathing solitons in direct simulations. Both methods reveal that stable solitons may be supported if the average value of the nonlinear coefficient (whose sign corresponds to attraction between atoms) and the lattice's strength exceed well-defined minimum values. Stable localized patterns may feature a multi-cell structure.
\end{abstract}

The creation of the Bose-Einstein condensates (BEC) in vapors of alkali metals has opened an opportunity to investigate nonlinear interactions of atomic matter waves. One of the most fundamental aspects of these studies is generation of solitons. Dark solitons had been created in effectively one-dimensional (1D) BEC with repulsive interatomic collisions [1]. Then, bright solitons were observed in 1D BECs with attractive collisions [2,3]. An issue of obvious interest is to develop methods for control of the matter-wave solitons. A promising approach consists in varying the scattering length (SL) of the interatomic collisions by means of an external magnetic field through the zero-SL point close to the Feshbach resonance [4]. In particular, it was shown that an abrupt change of the SL can result in splitting of a soliton into a set of secondary ones [5].

The application of ac magnetic field may induce a periodic modulation of the SL, opening a way to the "Feshbach-resonance management" (FRM) [6]. Quite a noteworthy FRM-induced effect is a possibility to create self-trapped oscillating BEC solitons (breathers) without any external trap. The underlying mechanism is balance between compression and expansion

$\left({ }^{*}\right)$ E-mail: mmatu@fuw.edu.pl

(c) EDP Sciences 
cycles, corresponding to the time intervals when the SL is, respectively, negative and positive. In fact, this possibility was first realized in terms of the transverse light propagation in a bulk layered nonlinear medium, where the Kerr coefficient alternates between positive and negative values [7]. The respective model is formally equivalent to the two-dimensional (2D) Gross-Pitaevskii equation (GPE), see Eq. (11) below, in which the SL is periodically modulated in "time" (analog of the propagation distance in [7]) not harmonically, but as a piecewiseconstant function. Then, the BEC model based on the GPE with the harmonic modulation of the SL was directly investigated in Refs. [8-10], with a conclusion that the FRM makes it possible to stabilize, without the use of an external trap, 2D breathers, but not the 3D ones. The absence of the stabilization in the $3 \mathrm{D}$ case was also concluded in the optical model considered in Ref. [7], where the 3D soliton would correspond to a spatiotemporal "light bullet".

A remarkable feature of these results is that the $2 \mathrm{D}$ breather is stable despite a possibility of the collapse in the 2D GPE with a negative SL. On the other hand, it has been recently demonstrated that solitons in BECs with a constant negative SL may be completely stabilized by means of an optical lattice (OL), i.e., a spatially periodic potential. It is true in both 2D and 3D cases [11]. Moreover, it was also shown that the 2D and 3D BEC solitons can be stabilized by low-dimensional OLs, i.e., respectively, by a quasi-1D (Q1D) lattice in the 2D case, and by a quasi-2D (Q2D) lattice in the 3D space [12]. However, 3D solitons cannot be made stable in a Q1D OL.

These results suggest a question whether 3D solitons can be stabilized by a combination of a Q1D lattice and FRM. The issue has practical relevance, as a Q1D OL can be easily created, illuminating the BEC by a pair of counterpropagating laser beams that form a periodic interference pattern [13]. This problem is different from that considered in Ref. [10], where stabilization of a localized 3D structure was provided by adding a tight $1 \mathrm{D}$ parabolic trap to the model, which made it nearly two-dimensional.

In this paper, we demonstrate that the combined OL-FRM stabilization of 3D solitons is possible indeed. First, we introduce the model and propose an experimental scheme. Then, we predict stability conditions for the 3D solitons by means of a variational approximation (VA). Finally, we demonstrate the soliton's stability in direct simulations. We also demonstrate that these results find another physical realization in nonlinear optics: a possibility to create a stable 3D "light bullet" (spatiotemporal soliton) in a bulk medium which combines an alternating self-focusing/defocusing structure in the longitudinal direction, and periodic modulation of the refractive index in one transverse direction.

The model. - The GPE for the single-particle wave function $\psi$, including a timedependent (FRM-controlled) nonlinear coefficient $g(t)$ and an external potential $V(\mathbf{r}, t)$, in normalized units is

$$
i \psi_{t}=\left[-(1 / 2) \nabla^{2}+V(\mathbf{r}, t)+g(t)|\psi|^{2}\right] \psi .
$$

We start by considering a BEC in the ground state of a radial (2D) parabolic trap with the (time-dependent) frequency $\omega_{\perp}(t)$, supplemented, in the longitudinal direction, by "end caps", induced by transverse light sheets. The configuration is like the one used to create soliton trains in the $\mathrm{Li}^{7}$ condensate [3]. Then, a 1D lattice in the axial direction, whose period is normalized to be $\pi$, is adiabatically switched on, by increasing its strength $\varepsilon$ from 0 at $t=0$ to a final value $\varepsilon_{\mathrm{f}}$ at $t=t_{2}$, see fig. 11 Thus, the full potential is

$$
V(\mathbf{r}, t)=\varepsilon(t)[1-\cos (2 z)]+(1 / 2) \omega_{\perp}^{2}(t) \varrho^{2}+V_{0}(z),
$$

where $\varrho$ is the radial variable in the plane transverse to $z$, and the axial end-cap potential $V_{0}(z)$ is approximated by a deep and wide potential box. 


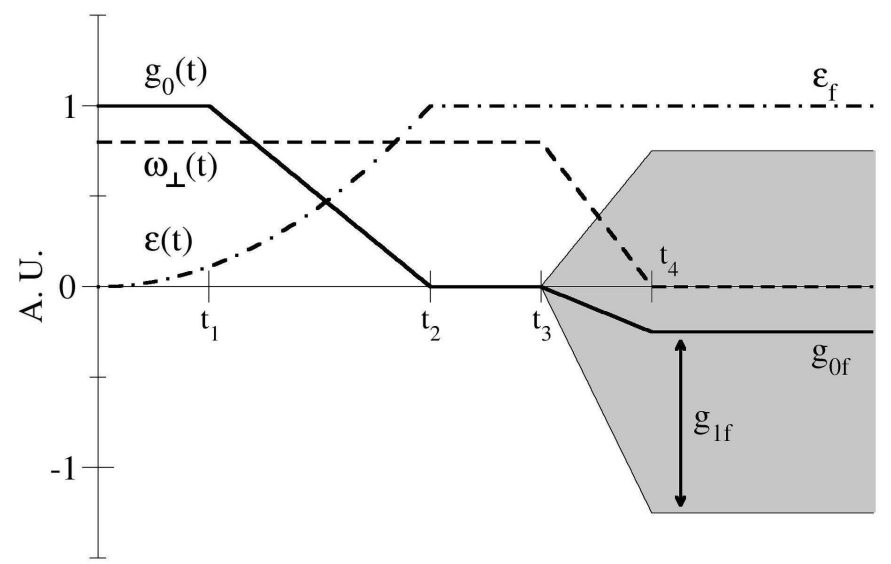

Fig. 1 - The time dependence of the nonlinear coefficient, $g$, transverse-confining frequency, $\omega_{\perp}$, and optical-lattice strength, $\varepsilon$, in the numerical experiment which leads to the establishment of stable $3 \mathrm{D}$ breathing solitons supported by the combination of the quasi-1D lattice and Feshbach-resonance management (FRM). The shaded area indicates rapid oscillations of $g$, which account for the FRM.

At some moment $t=t_{1}<t_{2}$, we begin to linearly decrease an initially positive nonlinear coefficient $g(t)$. It vanishes at the moment $t=t_{2}$, and remains zero up to $t=t_{3}$, when we start to gradually switch on the rapid FRM modulation of the nonlinear coefficient (in fig. [1 $g(t)$ is denoted as $g_{0}(t)$, up to $\left.t=t_{3}\right)$. At $t>t_{4}, g(t)$ oscillates with a constant amplitude $g_{1 \mathrm{f}}$ around a negative average value $g_{0 \mathrm{f}}$,

$$
g(t)=g_{0 \mathrm{f}}+g_{1 \mathrm{f}} \sin (\Omega t) .
$$

Finally, the radial confinement is switched off within the time interval $t_{3}<t<t_{4}$. After that, a soliton, if any, may only be supported by the combination of the Q1D lattice and FRM (the axial width of the established soliton is assumed to be much smaller than the distance between the end caps, hence they do not affect it).

Numerical experiments following the path outlined in fig. 10 make it possible to create stable 3D solitons. Before showing the results, we first resort to the VA, in order to predict conditions on the strength of the OL and size of the negative average nonlinear coefficient $g_{0 \mathrm{f}}$, which are necessary to support 3D solitons.

Note that the eventual form of Eq. (11), i.e., with $g$ and $V$ taken, respectively, as in Eqs. (3) and (2), where $\varepsilon$ is constant and $\omega_{\perp}=V_{0}(z)=0$, has an alternative physical interpretation in terms of nonlinear optics. Upon interchanging variables $t$ and coordinates $z$ (chosen to be a propagation direction) Eq. (11) describes pulse propagation in a bulk (3D) medium composed of alternating self-focusing and self-defocusing layers, similar to that introduced in Ref. [7], with an additional periodic modulation of the refractive index in the transverse direction $x$. Stable 3D solitons in the present model imply the existence of stable fully three-dimensional spatiotemporal solitons ("light bullets") in this medium. Thus far, only quasi-2D spatiotemporal solitons were created experimentally in bulk media [14].

Variational approximation. - The VA was successfully applied to the description of BEC dynamics under diverse circumstances, see, e.g., Refs. [5, 8-12,15] and a review [16]. 
Equation (11) is derived from the Lagrangian

$$
L=\pi \int\left[i\left(\psi_{t}^{*} \psi-\psi_{t}^{*} \psi\right)-\left|\psi_{\varrho}\right|^{2}-\left|\psi_{z}\right|^{2}-g(t)|\psi|^{4}-2 V|\psi|^{2}\right] \varrho \mathrm{d} \varrho \mathrm{d} z .
$$

As it is frequently done, we choose a variational ansatz for the solution based on the complex Gaussian with an amplitude $A(t)$, radial and axial widths $W(t)$ and $V(t)$ respectively, $b(t)$ and $\beta(t)$ being the corresponding "chirps"

$$
\psi(\mathbf{r}, t)=A \exp \left(-\varrho^{2}\left[1 /\left(2 W^{2}\right)+i b\right]-z^{2}\left[1 /\left(2 V^{2}\right)+i \beta\right]\right),
$$

The reduced Lagrangian is obtained by inserting the ansatz (5) into Eq. (4). The equations obtained by varying the reduced Lagrangian yield the conservation of the number of atoms, $E \equiv \pi^{-3 / 2} \int|\psi|^{2} \mathrm{~d} \mathbf{r}=A^{2} W^{2} V$, and dynamical equations for the widths,

$$
\begin{aligned}
\ddot{W} & =\frac{1}{W^{3}}-\omega_{\perp}^{2}(t) W+\frac{E g(t)}{\sqrt{8} W^{3} V}, \\
\ddot{V} & =\frac{1}{V^{3}}-4 \varepsilon V \exp \left(-V^{2}\right)+\frac{E g(t)}{\sqrt{8} W^{2} V^{2}} .
\end{aligned}
$$

A necessary condition for the existence of a $3 \mathrm{D}$ soliton in the present model can be derived from these equations in a crude approximation, cf. Ref. [8]. To this end, we assume that $g_{1 \mathrm{f}}$ in Eq. (3) is small, while $\Omega$ is large. It is also conjectured that the average value $\bar{W}$ of the soliton's radial size, $W$, is large (see below). Further, in the lowest approximation, the soliton's size in the axial direction may be assumed constant, $V(t) \approx V_{0}$, as determined by the relation

$$
4 \varepsilon V_{0}^{4} \exp \left(-V_{0}^{2}\right)=1,
$$

that follows from Eq. (6) where the last small term $\left(\sim W^{-2}\right)$ is dropped. Equation (8) has real solutions if the OL strength exceeds a minimum (threshold) value,

$$
\varepsilon \geq \varepsilon_{\mathrm{thr}}=e^{2} / 16 \approx 0.46 .
$$

At $\varepsilon>\varepsilon_{\text {thr }}$, Eq. (8) has two real solutions, which implies the existence of two different solitons. It seems very plausible (cf. Refs. $[11,12]$ ) that the narrower one, corresponding to smaller $V_{0}$, is stable, and the other one is unstable.

Next, replacing $V(t)$ by $V_{0}$ in Eq. (7) (we set $\omega_{\perp}=0$, to consider the possibility of the existence of the $3 \mathrm{D}$ soliton without the radial confinement) and substituting $g(t)$ from Eq. (3), we look for a solution as $W(t) \approx \bar{W}+W_{1} \sin (\Omega t)$. For large $\Omega$, the variable part of the equation

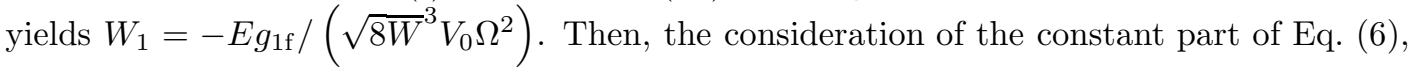
with regard to the first correction generated by the product of the oscillating terms in $W(t)$ and $g(t)$, produces a result,

$$
\bar{W}^{4}=\frac{3}{4 \sqrt{2} V_{0}}\left(\frac{E g_{1 \mathrm{f}}}{\Omega}\right)^{2}\left(E\left|g_{0 \mathrm{f}}\right|-\sqrt{8} V_{0}\right)^{-1} .
$$

An essential corollary of Eq. (10) is that a necessary condition for the existence of the 3D soliton is (recall $g_{0 \mathrm{f}}$ is negative)

$$
\left|g_{0 \mathrm{f}}\right|>\left(\left|g_{0 \mathrm{f}}\right|\right)_{\min }=\sqrt{8} V_{0} / E .
$$

Direct simulations presented in the next section show that this condition holds indeed, albeit approximately. Note that the above approximation, which assumed large $\bar{W}^{4}$, is valid only when $\left|g_{0 f}\right|$ slightly exceeds the minimum value defined in Eq. (11). 

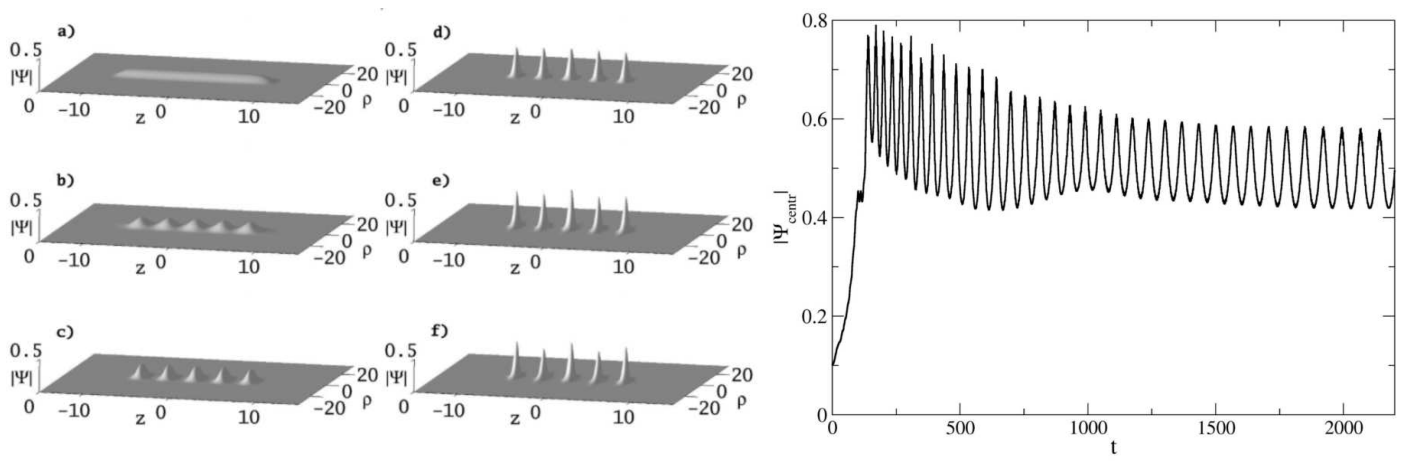

Fig. 2

Fig. 3

Fig. 2 - Evolution of the absolute value of the wave function in the pattern comprising five cells of the optical lattice. The normalized parameters are $g_{0 \mathrm{f}}=22.5, g_{1 \mathrm{f}}=4 g_{0 \mathrm{f}}, \epsilon_{\mathrm{f}}=25, \Omega=40, \omega_{\perp}(0)=0.3$, $t_{1}=30, t_{2}=100, t_{3}=120$, and $t_{4}=130$. Snapshots are taken at $t=0(\mathrm{a}), 20(\mathrm{~b}), 50(\mathrm{c}) 110(\mathrm{~d})$, 200 (e), 2000 (f).

Fig. 3 - Evolution of the central peak amplitude from fig. 2

Numerical results. - We simulated both the full GPE (Eq. 1) using an axisymmetric code, and the variational equations (6) and [7). In both cases we used Runge-Kutta methods. In the former case, the simulations followed the path outlined in fig. 1] Example of the numerical results, which is quite generic for strong lattice, is displayed in figs. 2 and 3, For instance. for ${ }^{87} \mathrm{Rb}$ atoms, the OL period is $\lambda=1.5 \mu \mathrm{m}$, the FRM and initial radial-confinement frequencies are, respectively, $\Omega=132 \mathrm{kHz}$ and $\omega_{\perp}(0)=990 \mathrm{~Hz}$, the lattice depth is $\varepsilon=25$ recoil energies, and the effective nonlinear coefficient is $n a= \pm 4 \cdot 10^{-6} \mathrm{~m}$, where $n$ is the number of atoms per the lattice cell, the total number of atoms being in the range of $10^{4}-10^{6}$. The respective values of the normalized parameters are given in fig. 2 caption.

In fig. 22 we show 3D snapshots of the wave-function pattern occupying five lattice cells, taken at times corresponding to various stages of the numerical experiment, cf. fig. 10 Comparison of the last two snapshots, (e) and (f), shows that condensate develops a robust structure, which remains unchanged over many FRM cycles.

Figure 3 displays evolution of the central-peak's amplitude in the same pattern. After an initial transient, the stable structure is established, featuring breathings without any systematic decay.

Actually, the stable pattern like the one shown in fig. 2 is a set of uncoupled fundamental solitons, each being trapped in a single lattice cell. This conclusion follows from additional numerical experiments, in which removal of the atomic population from any subset of the cells did not, in any tangible way, affect the localized states in other cells; in particular, the soliton sitting in a single cell is as stable as any multi-cell pattern. Thus, the conclusion is that the FRM may support a stable 3D soliton (breather) confined to a single cell of the Q1D lattice, and the solitons trapped in adjacent cells do not interact. In fact, this conclusion complies with the variational ansatz (5), which ignores any possible spatial oscillations induced by the OL, hence it indeed implies a soliton essentially confined to a single cell (cf. the VA for stationary models with the multi-dimensional OLs developed in Refs. [11] and [12]).

We have collected results of systematic GPE simulations and compared them with predictions of the VA based on simulations of Eqs. (6) and 17), as shown in fig. [4 As is seen in fig. 目(a), only the bottom part (corresponding to smaller values of the FRM frequency $\Omega$ ) of 


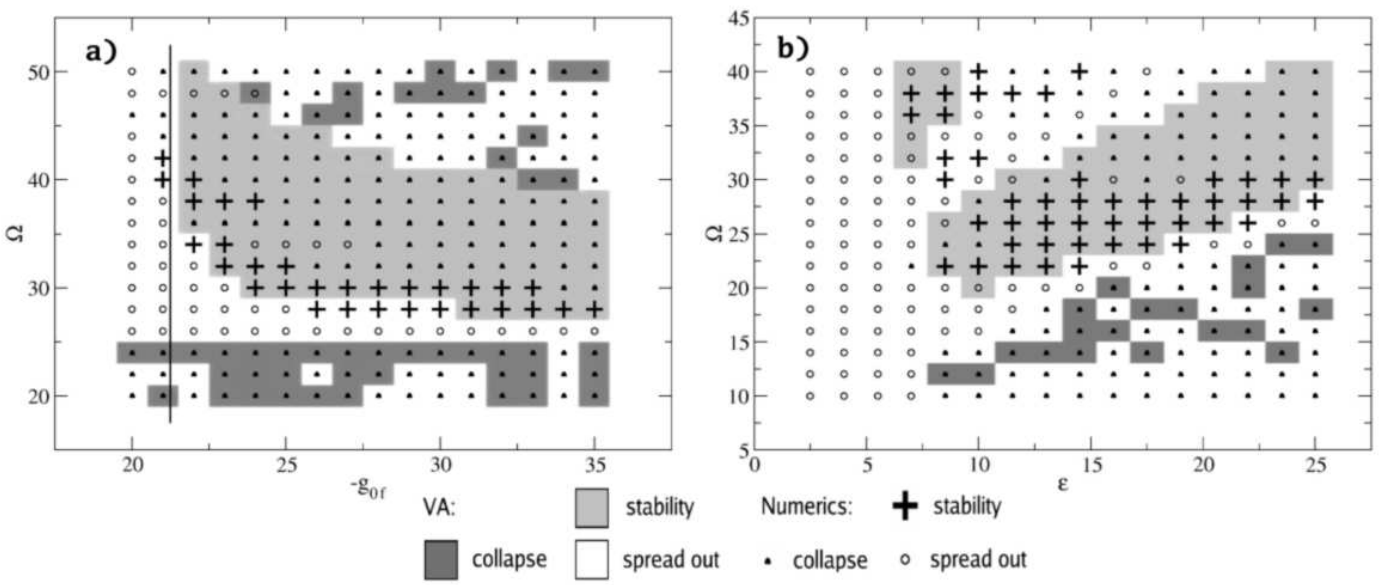

Fig. 4 - Stability regions for the 3D solitons as predicted by the variational approximation, and found from direct simulations of the Gross-Pitaevskii equation in (a) the $\left(g_{0 f}, \Omega\right)$ plane, and (b) the $(\epsilon, \Omega)$ plane. Other parameters are as in fig. 2 except that $g_{0 f}=30$ in (b). The vertical line in (a) corresponds to the minimum value of $\left|g_{0 f}\right|$ predicted by Eq. (11).

the VA-predicted stability region in the $\left(g_{0 \mathrm{f}}, \Omega\right)$ plane actually supports stable 3D solitons. The variational estimate (11) for the minimum size of the average nonlinear coefficient necessary for the existence of the 3D soliton in the Q1D lattice is borne out by the simulations, although approximately.

Lastly, the simulations definitely demonstrate the existence of a minimum of the OL strength $\varepsilon$ which is necessary to support the $3 \mathrm{D}$ solitons, as it was predicted by the VA, see Eq. (9). Figure 4(b) shows regions of stability in the $(\epsilon, \Omega)$ plane. From this figure we conclude that VA predicts stability regions quite well, but the numerical value of the $\varepsilon_{\text {thr }}$ is somehow larger than that predicted by the simple approximation given by Eq. (9). For simulations presented in figs. 2 and 3 we have chosen the value of $\varepsilon$ well above the threshold.

Conclusions. - In this work, we have proposed a scheme to stabilize 3D solitons in BECs where the interatomic interaction is attractive, on average. The scheme is based on the combination of the Feshbach-resonance management and quasi-1D optical lattice. In previous works, it has been shown that these two methods applied separately can stabilize 2D solitons, but not 3D ones. The combined method proposed here can be easily implemented in the experiment, and thus opens a way to the creation of 3D solitons in BECs. Another physical implication of the results is a stable 3D "light bullet" in an optical medium with an alternating longitudinal self-focusing/defocusing structure, and periodic modulation of the refractive index in a transverse direction.

The possibility to achieve the result was demonstrated within the framework of the variational approximation (VA) and verified in direct simulations. In particular, the VA predicts that the OL's strength and average value of the oscillating nonlinear coefficient must exceed certain minimum values. These predictions are indeed corroborated by the simulations. However, the full stability region predicted by the VA extends much farther into the highfrequency region than the actual stability area found in the direct simulations. The stable patterns found in the simulations may feature a multi-cell structure, which in the case studied here form a set of non-interacting fundamental solitons, each trapped in a single cell of the 
OL, in good qualitative agreement with the description provided by the VA. In weak lattices one may expect to observe interaction between individual solitons. This will be a subject of further investigation.

M.M. acknowledges support from the KBN grant 2P03 B4325, M.T. was supported by Polish Ministry of Scientific Research and Information Technology under the grant PBZ MIN008/P03/2003. The work of B.A.M. was partially supported by the Israel Science Foundation through the grant No. 8006/03.

\section{REFERENCES}

[1] Burger S. et al., Phys. Rev. Lett., 83 (1999) 5198; Denschlag J. et al., Science, 287 (2000) 97

[2] Strecker K. E. et al., Nature, 417 (2002) 153.

[3] Khaykovich L. et al., Science, 296 (2002) 1290.

[4] Inouye S. et al., Nature, 392 (1998) 151; Cornish S. L. et al., Phys. Rev. Lett., 85 (2000) 1795; Donley E. A. et al., Nature, 412 (2001) 295; Saito H. and Ueda M., Phys. Rev. A, 65 (2002) 033624.

[5] Carr L. D. and Castin Y., Phys. Rev. A, 66 (2002) 063602.

[6] Kevrekidis P. G., Theocharis G., Frantzeskakis D. J. and Malomed B. A., Phys. Rev. Lett., 90 (2003) 230401.

[7] Towers I. and Malomed B. A., J. Opt. Soc. Am. B, 19 (2002) 537.

[8] Saito H. and Ueda M., Phys. Rev. Lett., 90 (2003) 040403.

[9] Abdullaev F., Caputo J. G., Kraenkel R. A. and Malomed B. A., Phys. Rev. A, 67 (2003) 013605.

[10] Montesinos G. D., Perez-Garcia V. M. and Torres P. J., Physica D, 191 (2004) 193.

[11] Baizakov B. B., Malomed B. A. and Salerno M., Europhys. Lett., 63 (2003) 642.

[12] Baizakov B. B., Salerno M. and Malomed B. A., Nonlinear Waves: Classical and Quantum Aspects, edited by F. Kh. Abdullaev and V. V. Konotop (Kluwer: Dordrecht) 2004, p. 61; Baizakov B. B., Salerno M. and Malomed B. A., Phys. Rev. A, in press.

[13] Jurczak C. et al., Phys. Rev. Lett., 77 (1996) 1727; Stecher H. et al., Phys. Rev. A, 55 (1997) 545; Jaksch D. et al., Phys. Rev. Lett., 81 (1998) 3108; Marzlin K. P. and Zhang W. P., Phys. Rev. A, 59 (1999) 2982.

[14] Liu X., Qian L. J. And Wise F. W., Phys. Rev. Lett., 82 (1999) 4631; Liu X., Beckwitt K. and Wise F., Phys. Rev. E, 62 (2000) 1328.

[15] PÉrez-García V. M. et al., Phys. Rev. Lett., 77 (1996) 5320; PÉrez-García V. M. et al., Phys. Rev. A, 56 (1997) 1424; Ueda M. and Leggett A. J., Phys. Rev. Lett., 80 (1998) 1576; Menotti C., Anglin J. R., Cirac J. I. and Zoller P., Phys. Rev. A, 63 (2001) 023601; Trombettoni A. and Smerzi A., J. Phys. B, 34 (2001) 4711; Ohberg P. and Santos L., J. Phys. B, 34 (2001) 4721; Alexander T. J. and Bergé L., Phys. Rev. E, 65 (2002) 026611; Kevrekidis P. G. et al., J. Phys. B, 36 (2003) 3467; Abdullaev F. K. and Salerno M., J. Phys. B, 36 (2003) 2851; Rapti Z., Kevrekidis P. G., Smerzi A. and Bishop A. R., Phys. Rev. E, 69 (2004) 017601; Abdullaev F. K., Gammal A. and Tomio L., J. Phys. B, 37 (2004) 635; Salasnich L., Parola A. and Reatto L., Phys. Rev. A, 69 (2004) 045601; 70 (2004) 013606; Jackson A. D., Kavoulakis G. M. and Lundh E., Phys. Rev. A, 69 (2004) 053619; Pérez-García V. M., Physica D, 191 (2004) 211.

[16] Malomed B. A., Progr. Opt., 43 (2002) 69. 